\title{
A Self-organization Analysis of Knowledge Collaboration with Enterprise 2.0 Application
}

\author{
Xue Yun and Chen Jianbin ${ }^{1}$ \\ Business College of Beijing Union University, Beijing, China \\ Yun.xue@buu.edu.cn, jianbin.chen@buu.edu.cn
}

\begin{abstract}
The development of Web 2.0 and its social application lead to the appearance of Enterprise 2.0. Based on the discussion of self-organization of enterprise knowledge system in Enterprise 2.0, this paper built a super-network model integrated the interpersonal network and the knowledge object network as well as the definition of the super-network structure entropy. Take the knowledge governance as a regulator variable between enterprise knowledge system and its environment, a relationship between the performance of knowledge collaboration and structure entropy has been built, then a Logistic Growth Model has been proposed to analysis the self-organization process.
\end{abstract}

Keywords: Enterprise 2.0, Knowledge collaboration, self-organization, super-network model

\section{Introduction}

In an enterprise knowledge system, information flows in a narrow channel traditionally (McAfee, 2011). According to the limit capability of information and knowledge processing of any single node in this channel, the knowledge transfer may be paused or delayed. A reasonable inference is that, there may be very important information or knowledge had not been transferred appropriately in time. Former researches on knowledge management normally focus on the form exchange, influencing factors and management measures in the knowledge processing. The basic research modes are also restricted to the channel structure. A new mode, which studied in this paper, collaboration knowledge processing have not been supported adequately by technology and theory.

To put the right information and knowledge to the right people at right time and right site, is the goal of knowledge collaboration research. There are many successful social collaboration cases in web 2.0 environments, such as FACEBOOK, Wikipedia and so on, which are all typical knowledge collaboration pattern. Accompany with the wide application of web 2.0 technologies in enterprise Intranet or Extranet, enterprise knowledge collaboration which so called Enterprise 2.0(E2.0) is becoming our hot topic now.

${ }^{1} *$ Corresponding author: Chen Jianbin 


\section{Literature Review}

\subsection{Enterprise 2.0 Application and Its Research}

All Enterprise 2.0 as a concept subsumes the efforts of adopting social software, originating from the public Internet, for use in enterprise contexts and for professional purpose (McAfee 2006). McAfee defines Enterprise 2.0 as "the use of emergent social software platform within companies, or between companies and their partners of customers". E2.0 isn't just about applying social software, but it describes a wider approach that advocates a new culture of participation, inclusion, and sharing. Researches in this domain are most focus on Mashup system platform (Michele, et al., 2010), governance structure (Mckeley, et al., 2003) and their application. In China, the advantages of Web 2.0 technology had been discussed, and the characteristics of highly interaction, openness, flexibility, applicability and integration of web 2.0 have been considered as powerful weapon for knowledge acquisition, knowledge organization and sharing, then fostered the implementation of Knowledge Management (KM) system.

Enterprise 2.0 oriented to agile cooperation and knowledge sharing, advocate bottom-up management idea, decentralized management mode, flatter organization structure and widely customer participation. So it has a quite excellent capability widely used to acquire tacit knowledge, best practice and relevant experience in enterprise. To applied E2.0 into KM, those faults, such as limited usage of knowledge resource, low participation of whole staff, slow knowledge renewal, lack knowledge exchange and difficulty to meet personal requirement, could be make up in KM practice(Wang, et al., 2007). Otherwise, E2.0 is only a technology application pattern, and technology alone will not guarantee an organizational success for any investment in IS. Realizing the objectives of a software implementation depends heavily on how the organization and its constituents will interact with the given technological artifacts and sustain the use thereof within the fabric of the enterprise. It is well established in the IS literature that the most ambiguous, yet critical part of realizing the aspired benefits from an IT investment is providing for the right organizational complements to the technology(McAfee,2006). So, there are many works to do in knowledge governance and management layer.

\subsection{Knowledge Collaboration}

Karlenzig (2002) proposed the concept of Knowledge Collaboration (KC) at first. $\mathrm{He}$ considered it as a strategic organizational approach that dynamically builds upon internal and external systems, business processes, technology and relationships (communities, customers, partners and suppliers) to maximize business performance. $\mathrm{KC}$ demonstrates the extent to which a corporation has institutionalized processes for knowledge creation, capture, sharing and reuse as a fundamental means of creating value. These capabilities produce the greatest value when they are embedded in the fabric of an organization's culture, values, processes and reward systems. Corporations that want to succeed in the networked economy need to master knowledge collaboration. After that, other scholars defined $\mathrm{KC}$ as a knowledge activity (Mckelvey et al., 2003), organization capability (Glogel et al., 2006) or management mode and strategy means(Fan,2007) at the firm level, and as a special relationship pattern at the industry or supply chain level (Yang Lijun, 2011). Tong (2011) defined KC as a multidimensional dynamic process, in which all these factors, involves subject, object, environment of KM, interact to a highly synergy situation, so that the right knowledge or information can be transferred to right object just in time. So it is a highly 
development phase of $\mathrm{KM}$ to integrate knowledge resources and flows. Based on the definition of $\mathrm{KC}$, most researches in China focus on the process analysis of $\mathrm{KC}$ up to now (Table 1). And with this table, we can conclude as follows: firstly, $\mathrm{KC}$ aims at knowledge creation, that's means there should be new knowledge produced at the end of KC. Secondly, KC contains many kinds of knowledge transforms, such as knowledge sharing, transfer, internalization and externalization. Thirdly, in the process of KC, there are sub-processes like knowledge searching, knowledge transfer and creation.

Table 1. Literature Review of KC Process Study in China

\begin{tabular}{|c|c|c|}
\hline Author & Process of $\mathrm{KC}$ & Remark \\
\hline $\begin{array}{l}\text { Zen Deming, } \\
\text { WenXiaoke et } \\
\text { al,(2010) }\end{array}$ & $\begin{array}{l}\text { Knowledge achievement, transfer and creation } \\
\text { to get new knowledge }\end{array}$ & $\begin{array}{l}\text { Supply } \\
\text { chain level }\end{array}$ \\
\hline $\begin{array}{l}\text { Wang } \\
\text { Congying,Guan } \\
\text { Xiaodong }(2009) \\
\end{array}$ & $\begin{array}{l}\text { A closed-loop process of discovery, innovation, } \\
\text { dissemination, and re-discovery. }\end{array}$ & $\begin{array}{l}\text { Industry } \\
\text { cluster }\end{array}$ \\
\hline $\begin{array}{l}\text { Tong Zehua } \\
(2012)\end{array}$ & $\begin{array}{l}\text { Contains requirement of } \mathrm{KC} \text {, define subject of } \\
\mathrm{KC} \text {, activity of } \mathrm{KC} \text {, achievement of } \mathrm{KC} \text { and so on }\end{array}$ & $\begin{aligned} \text { Base on } \\
\text { process }\end{aligned}$ \\
\hline $\begin{array}{l}\text { Shi Huibin } \\
(2008)\end{array}$ & $\begin{array}{l}\text { A 4-tuple process model of KC: } \\
\text { environment,activities(relevant, reconstitution, } \\
\text { integrate, collide, interact, share), knowledge } \\
\text { flows (transfer/transform, internalization/ } \\
\text { Externalization) and Goals }\end{array}$ & \\
\hline $\begin{array}{l}\text { Wu Shaobo et } \\
\text { al(2008) }\end{array}$ & $\begin{array}{l}\text { Knowledge sharing/transform/KM/knowledge } \\
\text { creation }\end{array}$ & \\
\hline Wang Yue(2009) & $\begin{array}{l}\text { The core processes of } \mathrm{KC} \text { are knowledge } \\
\text { achievement, discovery, } \\
\text { dissemination and sharing, use and innovation. }\end{array}$ & \\
\hline
\end{tabular}

KC needs highly technology supportment, such as online knowledge-database or CSCW, to support its running (Lauric et al., 2006; Anklam, 2002; Tian, 2003). Zhan et al. (2002) constructed collaboration knowledge management system based on network for staff and customers to exchange/sharing knowledge in distributed space. Chen (2005) considered that Wikipadia is a whole new platform for cooperate work and knowledge sharing in Internet era. Su (2006) introduced collaboration concept to knowledge innovation, and thinks that BLOG can be a new platform for knowledge innovation. It is obvious that the emergent of web 2.0 application pushed up KC practice as well as relevant research in management theory.

\subsection{Research of Enterprise Knowledge System and Its Self-organization}

$\mathrm{KC}$ is an evolution status of knowledge system. Research demonstrated that knowledge, as the main factor of enterprise knowledge system, has bionic properties (He, 2008), and enterprise knowledge system is an activity system with biological feature. This kind of system has anti-entropy increase mechanism which can improve degree of order to be a highly order structure. A knowledge-based organization is a 
typical self-organization system with attributes of openness, non-equilibrium, nonlinear and fluctuations (Peng, 2005). Through the evolution process of enterprise system, external or heterogeneity knowledge can decrease system entropy and put system to a higher order structure.

Wikipedia and Baidu-Baike are self-organizational systems in web 2.0 era (Dan \& Zhao, 2009; Song et al., 2010). Enterprise wikis, microblog and virtual community in E2.0 application are main parts of enterprise knowledge system and have web 2.0 applied attributions, so they are self-organization knowledge systems. Voluntary cooperation can produce in E2.0 environment. If a demander has knowledge requirement, this information may be spread through web 2.0 platform among social network; a provider who own right knowledge for this requirement and fortunately in this network could meet this demand depend upon his interest. Based on the E2.0 platform, the self-organization feature of enterprise knowledge system makes an openness, dynamic and constant evolution knowledge forest for knowledge staffs to find problem, to analysis and solve it with rich and widely communication. So to speak that E2.0 enables staff to turn potential relationship of knowledge cooperation to real relationships and make tacit knowledge transfer into visualization knowledge collaboration.

But how can the enterprise knowledge system achieve synergy effect to fit organization strategy goals in E2.0 environment? Different with Wikipedia and BLOG on World Wide Web, E2.0 as a concept subsumes the efforts of adopting social software, origination from the public Internet, for use in enterprise contexts and for professional purposes (McAfee 2006). As the social software phenomenon is very closely connected to the unique culture of user participation and bottom up emergence typical for Web 2.0, transferring the phenomenon from the public Internet to the corporate context requires a good understanding of the phenomenon and its manifestation in different technical platforms. We will refer to the phenomenon of SNS (Social Network System)-base social networking in enterprise contexts as Enterprise Social Networking (ESN), a "Enterprise + social network" paradigm. In this article, a super-network model which comprise of interpersonal network and knowledge-object network should be constructed to represent the Enterprise Knowledge Network (EKN) with defined network structure entropy to measure system-order. Then a selforganization analysis has been carried out based on the entropy model and gotten some conclusions valuable for enterprise KM.

\section{The Super-network Structure of EKN and Its Order Measurement}

\subsection{The Super-network Structure of EKN}

Karl Popper had proposed three worlds theory: World 1(Objective World), being the physical world, or physical states; World 2(Subjective World), being the world of mind, or mental states, ideas, and perceptions; and World 3(Knowledge World), being the body of human knowledge expressed in its manifold forms, or the products of the second world made manifest in the materials of the first world (i.e., books, papers, paintings, symphonies, and all the products of the human mind). There are some cognition relations among three worlds, indicated in Figure 1. 


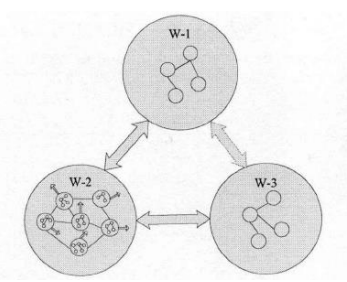

\section{Figure 1. Mapping Relations of World 1, 2, 3}

KM thinks that Enterprise Knowledge System contains all those knowledge factors around human resource in enterprise. There are mainly two kinds of factors, one is staffs corresponding to World 2(W-2) and another is knowledge objects expressed in vary medias (such as Enterprise BLOG, wiki, or virtual forum) corresponding to World 3 (W-3). W-2 produced W-3, which is the explicit expression of the knowledge collaboration result produced by individuals. Conversely, W-3 affected the evolution of relationship and structure of human network in W-2. The self-organization process of knowledge system reflects the co-evolution process of W-2 and W-3. Therefor a collaboration model combined two layer networks of W-2 and W-3 under the definition of super-network system with interpersonal network and knowledge objects network should be proposed as below.

Let $S$ denotes this super-network, $N_{k}$ is the network set in which $k=1,2$, then $S=$ $\left(N_{1}, N_{2}\right)$ means there are two network in this super-network, which are interpersonal network $N_{1}$ and knowledge objects network $N_{2}$ respectively.

Definition 1 Each network $N_{k}(k=1,2)$ in super-network $S$ can be expressed as $G_{k}=$ $\left(V, E_{k}, L_{k}\right)$, in which $V$ is a node set contained all nodes in $S$ and $E_{k}=\left(e^{k}{ }_{i j}\right)$ is an edge set contained all edges in $S . L_{k}=\left(l_{i j}^{k}\right)(i=1,2, \ldots, n ; j=1,2, \ldots, n)$ are weights of edge $\mathrm{e}^{\mathrm{k}}{ }_{\mathrm{ij}}$ in $N_{k}$, which means the weight of information flow in network.

Flows in network can be quantified in vary ways base on network characteristic. Normally, the edges are un-weighted while researching on knowledge transfer among interpersonal network based on SNA. In this case, edges may be only reflect whether there are relations between two neighbor nodes but have no information about relation strength of knowledge transfer. Our super-network model has an important characteristic which made up this disadvantage by statistical analysis based on knowledge objects network $N_{2}$.

\subsection{Definition of Network Structure Entropy}

From the research achievement on self-organization evolution of knowledge network in web 2.0 environment, it is an ordering path, which is from un-order to ordered, from equilibrium state to un-equilibrium state, with state mutation or emergence by accidently, for a knowledge system evolution. In the system theory, entropy is an antithesis concept of degree of order, which is regarded as the core index of system evolution providing a measurement possibility of the degree of order. Hence, we should define the network structure entropy to express the evolution state of enterprise knowledge system.

Abstract the network as graph $G=(V, E, L)$, in which $V$ is a node set, and $E$ is an edge set, as well as $L(i, j)$ represent an edge from node $i$ to node $j$. Consulted the definition of network structure entropy from articles of Tan(2004), we define the important degree of node $i$ or edge $j$ as 


$$
\mathrm{I}_{i}=\frac{k_{i}}{\sum_{i=1}^{N} k_{i}} \quad \mathrm{~J}_{j}=\frac{L_{j}}{\sum_{j=1}^{M} L_{j}}
$$

In which, $N$ is the number of node, $M$ is the number of edge, $k_{i}$ is connectivity of node $i$, and $L_{j}$ is the weight of edge $j$.

Then we can define Network Structure Entropy (NSE) as

$$
E=-\left(\sum_{i=1}^{N} I_{i} \ln I_{i}+\sum_{j=1}^{M} L_{j} \ln L_{j}\right)
$$

Regardless of enterprise knowledge object network such as BLOG and wiki, or interpersonal network, higher degree of knowledge network order means more effective of knowledge transfer or sharing, more possibility to achieve knowledge collaboration. With the super-network $S$ of knowledge system, We appoint $E_{l} 、 E_{2}$ and $E_{S}$ as the NSE of interpersonal network, knowledge object network and super-network itself respectively. If we can calculate all these NSEs on varies moment, then the degree of order of whole network, and change state of interpersonal network or knowledge object network should be measured more accurately.

\section{Self-organization Analysis of Enterprise Knowledge Collaboration}

\subsection{The Self-organization Analysis Model of Enterprise Knowledge System}

Relative to biology self-organization, it is mostly a domestically driven process for economic self-organization. The conscious and proactive behaviors of economic system help itself to gain new knowledge and techniques, to protect it from system inertia, such as energy/entropy constraint or history constraint. In knowledge system, this kind of conscious and proactive behavior can be called knowledge governance, which on behalf of conscious knowledge actions simulated and induced from factors outside, such as pressure of competitors, progress of basic science and change of law system. Knowledge governance are those actions, such as building knowledge exchange environment, setting up knowledge share culture and formulating knowledge transfer excitation mechanism, can affect relations between $\mathrm{W}-2$ and $\mathrm{W}-3$ to promote knowledge ecological system development. Thinking super-network as knowledge system itself, knowledge governance is a kind of regulation power acted on the system and determined the self-organization evolution process.

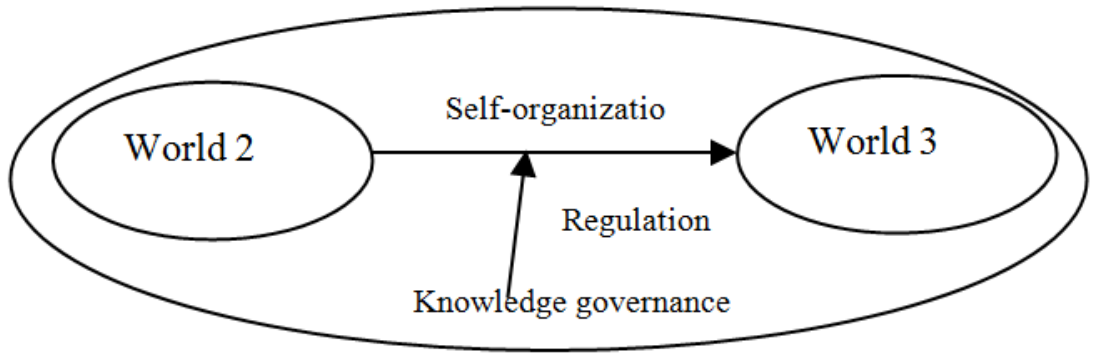

Figure 2. Enterprise Knowledge System Self-organization and Governance

Assume that $H=\left(H_{i}, \quad i=1,2, \ldots, n\right)$ as an action set of knowledge governance, which mainly are systems, rules and orders. The structuring or ordering process reflected in the value change of NSE. Generally, knowledge governance results the entropy 
decrease of knowledge system to offset the entropy increase resulted by knowledge system itself, then the entropy of whole system is reduced. Suppose the knowledge entropy equation of openness system as

$$
R=R_{H^{-}} R_{k}
$$

In which $R_{k}$ is the knowledge entropy produced by system itself and normally is positive value, $R_{H}$ is the knowledge entropy produced by interaction of system and its environment and reflect the affection degree of knowledge governance applied to knowledge subject's motivation and behavior. $R$ is the structuring degree of enterprise knowledge system, which can be measured by calculating NSE $E$ of super-network $S$.

Meanwhile, define $P$ as the performance of enterprise knowledge collaboration which reflects the matching degree of knowledge system structuring degree and enterprise performance currently, as well as the achievements of knowledge governance. Then $P$ can be considered as the function of knowledge entropy:

$$
\mathrm{P}=F(R)=F(E)
$$

\subsection{Self-organization Analysis of Enterprise Knowledge Collaboration}

An endogenous structuring process should be impossible a deterministic convergence process towards a stable equilibrium state (Kurt,2011). Prigogine \& Stengers (1984) point out that if we adopt a growth homogenous measure to measure structuring process, then the endogenous structuring process should be present as a special trajectory with time gone. This trajectory can be indicated by logistic diffusion equation which present as $S$ shape and will approach to static state on boundary.

4.2.1. The Logistic equation of Enterprise Knowledge System Evolution: The Logistic equation is a deterministic math equation which usually be used to express relationships among economic variables and to distinguish all kinds of powers acted on the system exchange process from a static state to a new static state. But the selforganization process is a kind of unbalanced endogenous process, and the trend of approach to instable structure can't be exactly reflected in Logistic function. However, if the research goal is not a deduction and shouldn't have any basic stable evaluation, Logistic equation still can provide abstractive expression for structuring in a smooth phase. Enterprise knowledge system is a kind of economic system, contains all those features of economic system evolution in its self-organization. In order to describe the process for enterprise knowledge system from an equilibrium state to a new one, as well as the fluctuation or emergence characteristics beside the tipping point, we adopt Logistic equation in this paper to identify regularity of knowledge collaboration.

Considered of the normal form of Logistic equation, let $X$ be structure variable, such as NSE of knowledge system, which will evolutions constantly over time:

$$
\mathrm{X}_{t}=X_{t-1}\left[1+b\left(1-\frac{X_{t-1}}{K}\right)\right]+u_{t}
$$

In which $b$ is diffusion ratio or structure ratio, and $K$ is load capabilities and $u_{t}$ is deviation. The change of structure ratio in a unit of time from Equation (5) should be:

$$
\frac{X_{t}-X_{t-1}}{X_{t-1}}=b-b \frac{X_{t-1}}{K}+\frac{u_{t}}{X_{t-1}} \quad \text { or approximately: } \ln X_{t}-\ln X_{t-1}=b-b \frac{X_{t-1}}{K}+e_{t}
$$




$$
\begin{aligned}
& \qquad e_{t}=\frac{u_{t}}{X_{t-1}} \text { 。 Equation (6) can be further extended as : } \\
& \text { In which, } \\
& \ln X_{t}-\ln X_{t-1}=[b(\ldots)]\left[1-\frac{X_{t-1}}{K(\ldots)}\right]+e_{t}
\end{aligned}
$$

In which $b$ and $K$ also become functions of other variables. Function $b(\ldots)$ contains various factors affected on diffusion ratio and is no longer a constant over time. Function $K(\ldots)$ then consider various factors which can extend or contract the load capability of knowledge system. Generally, factors contained in Function $b(\ldots)$ have short influence on economic system and factors in Function $K(\ldots)$ have long influence which is deterministic on structuring.

The possibility of unstable and discontinuous structure transform of Knowledge system while it get into the structuring stage of Logistic curve. It is helpful for economic self-organization analysis to endogenous the basic parameters of logistic equation.

4.2.2. Government means of Logistic Equation: There are two important problems should be considered while researching on collaboration evolution of enterprise knowledge system, one is what factors will affect the diffusion ratio or structuring ratio, and another one is what factors will affect the tipping point of structuring. Which factor can be contained into function $b(\ldots)$ and $K(\ldots)$ should be determined by the existence of knowledge evaluation, exchange and contract.

According to economic theory, short term factors mainly are economic and measurable, such as relative price and flow of knowledge contained in function $b(\ldots)$, and long term factors mainly are uneconomic and not to be measured, such as variable in layer of system, culture and mentality of enterprise knowledge system contained in function $K(\ldots)$. Short term factors will affect the diffusion ratio of system in the near future, but the movement of unmeasurable variables like the change of governance structure or supervision system, will have deterministic effect on structuring process in long time. Through research on system, those important changes and its happen time should be identified and should be expressed as different qualitative movement variable.

Equation (7) provides a possibility to quantitative observe the evolution process of enterprise knowledge system. Through data calculating, the logistic relationship can be confirmed so that basic logistic form can be separated from it and which structuring stage the knowledge system stayed in also can be identified. Based on these determinations, the stability of knowledge system structure can be assessment while outside impact happened such as knowledge lash resulted from dramatic change of competitive environment. When knowledge system developed into the saturation stage of logistic curve, its structure will face the challenges of unstable or structure interrupt. It is helpful to study on these features of this stage for enterprise to execute necessary measures to avoid key knowledge oscillation caused by structure interrupt and adjust policies guiding knowledge system evenly transmit to new stage.

\section{Conclusion}

It is not so effective for traditional knowledge management to manage tacit knowledge (Yan et al., 2001). But E2.0 has taken new opportunity for knowledge 
collaboration because of its more powerful capability to solve practice problems with tacit knowledge. In this paper, we constructed the self-organization concept model and logistic process analysis model of knowledge system in E2.0 environment, which are to explore the evolution pattern of knowledge collaboration system in favor of the organization mechanism design for enterprise knowledge governance. In future, there are some topics should be discussed, including the simulation calculation of knowledge system evolution in E2.0 environment, searching for the upper limit, lower limit and optimal value, presumption appropriate control parameters and formulation of governance structure and strategies.

\section{Acknowledgments}

The authors gratefully acknowledge the Funding project of research plan from the Education Committee of Beijing (SQSM201211417002), the Funding Project for Academic Human Resources Development in Beijing Union University (BPHR2012A02) and the Funding Project for Academic Human Resources Development in Institutions of Higher Learning Under the Jurisdiction of Beijing Municipality (No. PHR201008332).

\section{References}

[1] A. P. McAfee, "Mastering the three worlds of information technology", Harvard Business Review vol. 84 , no. 11, (2006), pp. 141-149.

[2] P. Anklam, "Knowledge management: the collaboration thread", Bulletin of the American Society for Information Science and Technology, vol. 6, (2002), pp. 8-11.

[3] D. Jinhua and P. Zhao, "The Phenomenon of Knowledge Self-organization Based on the Baidu Known and Its Enlightenment", Sci-Tech Information Development\& Economy, vol. 2, (2009), pp. 114-116.

[4] Z. Fan, B. Feng and Z. Yu, "The Developing and Research Prospects for Knowledge Collaboration", Science of Science and Management of S \& T, vol. 11, (2007), pp. 85-91.

[5] J. He and J. Li, "A biological model of knowledge development", Studies in Science of Science, vol. 26, no. 4, (2008), pp. 679- 684.

[6] M. Swift, D. B. Balkin and Matusik, „Goal Orientations and the Motivation to Share Knowledge”, Journal of Knowledge Management, vol. 3, (2010), pp. 378-393.

[7] M. Mckelvey, H. Almb and M. RiccaboniI, "Does co-location matter for formal knowledge collaboration in the Swedish biotechnology-pharmaceutical sector", Research Policy, vol. 32, (2003), pp. 483-501.

[8] J. Peng and B. Zhao, "On the Theory of Self-organization and Knowledge Management", Journal of Systemic Dialectics, vol. 1, (2005), pp. 37-41.

[9] Q. Song, "Comparative analysis of network structure between self-organization knowledge system and organized knowledge system", Information Studies: theory \& Application, vol. 3, (2010), pp. 115-119.

[10] S. Zhen, "Behavior Analysis of Synergistic Knowledge Innovation Based on BLOG", Information Science, vol. 6, (2006), pp. 910-914.

[11] Y. Tan and J. Wu, "Network Structure Entropy and Its Application to Scale-free Networks", Systems Engineering-theory \& Practice, vol. 6, (2004).

[12] T. Feng, R. Li, X. Gu, M. Qin and J. Zhang, "Study of Management of Knowledge", Human-resource and Organization in Collaborative Design, Systems Engineering-theory \& Practice, vol. 7, (2003), pp. 17-23.

[13] T. Zehua, "Analysis of knowledge collaboration connotation”, Information Studies: Theory \& Application, vol. 11, no. 34, (2011), pp. 11-15.

[14] T. Zehua, "Knowledge Collaboration (KC) and the Relationship between KC and Some Related Concepts", Library and Information Service, vol. 56, no. 8, (2012), pp. 107-112.

[15] W. Wang, R. Xiong and J. Cheng, "Constructing Web2.0-based Knowledge Management Platform by DotNetNuke", New Technology of Library and Information Service, vol. 7, (2007). 
[16] S. Wu and X. Gu, "Research on the Knowledge Synergy of Knowledge Chain's Inter- firm Cooperation", Science of Science and Management of S \& T, vol. 8, (2008), pp. 83- 87.

[17] N. Yan, L. Sun and H. Li, "From Knowledge Organization to Knowledge Self- organization", Information Science, vol. 7, (2001), pp. 766-769.

[18] L. Yang, "Analysis on the Function of Supply Chain Knowledge Collaboration to Competition of Enterprises", Science and Technology Management Research, vol. 5, (2011), pp. 173- 175.

[19] H. Zhan, W. B. Lee and X. Gu, "Research on the Networked Collaborative Knowledge Management System", Computer Engineering and Applications, vol. 14, (2002), pp. 28-30.

[20] D. Zeng, X. Wen and Q. Chen, "Study on the mechanism of supply chain knowledge stock of enterprise growth based on knowledge collaboration", Forum on Science and Technology in China, vol. 2, (2010), pp. 77-81.

[21] I. Prigogine and I. Stengers, "Order Out of Chaos: Man's New Dialogue with Nature”, Boulder, CO, and London: New Science Library and Heinemann, (1984).

[22] A. P. McAfee, "Enterprise 2.0: New Collaborative Tools for Your Organization's Toughest Challenges, Beijing, China Machine Press, (2011), pp. 27-29.

[23] K. Dopfer, "The Evolution Basic of Economics", Beijing, Peking University Press, (2011), pp. 330-334.

[24] W. Karlenzig, "Tap into the Power of Knowledge collaboration [EB/OL]", Dimension Data, httP://www.tmcnet.com/, (2002).

[25] G. Laurie, H. Paula, H. Harlan, L. Robert, M. Robert and M. Ron, "Knowledge collaboration for IT support", available at httpt://www.helPdeskinstn/hdi2006/files/Strategic Adysory Board Paper Knowledge Collaboration.pdf. (2006).

[26] H. Shi, "Study on the concept analysis and psychological contract knowledge collaboration", Shen Yang, Northeastern University, (2008).

\section{Authors}

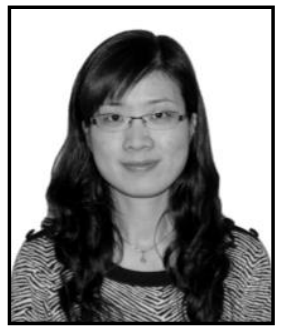

Xue Yun(1977- ), she is an Associate Professor of Computer Science at Beijing Union University, focus on Business Intelligence and Information Management.

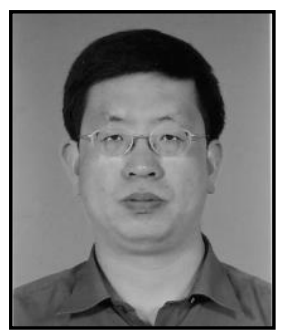

Chen Jianbin(1970- ), he is Professor of Information management at Beijing Union University, holds a master degree(1996) of system engineering and a doctor degree (2005) of Computer Application. Recent years, he observes his interest on Knowledge Management or Knowledge Collaboration $(\mathrm{KC})$, had published several journal articles and a book about KC. 